\title{
EDITORIAL
}

\section{Celebrating our first 10 years}

T his month marks the $10^{\text {th }}$ anniversary of the launch of Nature Reviews Nephrology. Such an occasion gives us cause to reflect on how the field of nephrology has progressed in the past decade and speculate as to what advances we might expect or hope to see in the future. This theme, of looking at past achievements and considering what the future might hold, is the focus of our anniversary issue.

In his editorial in the first issue of the journal (then called Nature Clinical Practice Nephrology), the inaugural Editor-in-Chief, Robert Schrier, described the formidable challenge of keeping abreast of the "myriad advances in cellular and molecular medicine." He outlined how the journal aimed to address this challenge by selecting, interpreting and presenting information with the greatest clinical implications in a uniquely digestible format. The past decade has witnessed an unprecedented evolution in academic publishing and an immense growth of digital and social media platforms. Nature Reviews Nephrology has also undergone some notable changes-for example, we now provide metrics for each article and use a dedicated Twitter account to engage with readers. But, in an era in which we are continuously inundated with information, our mission remains unchanged: to act as a filter to help busy clinicians and researchers keep up to date with the latest basic and clinical nephrology research through the provision of informative and accessible Reviews and commentaries.

An official journal of the International Society of Nephrology (ISN) since launch, Nature Reviews

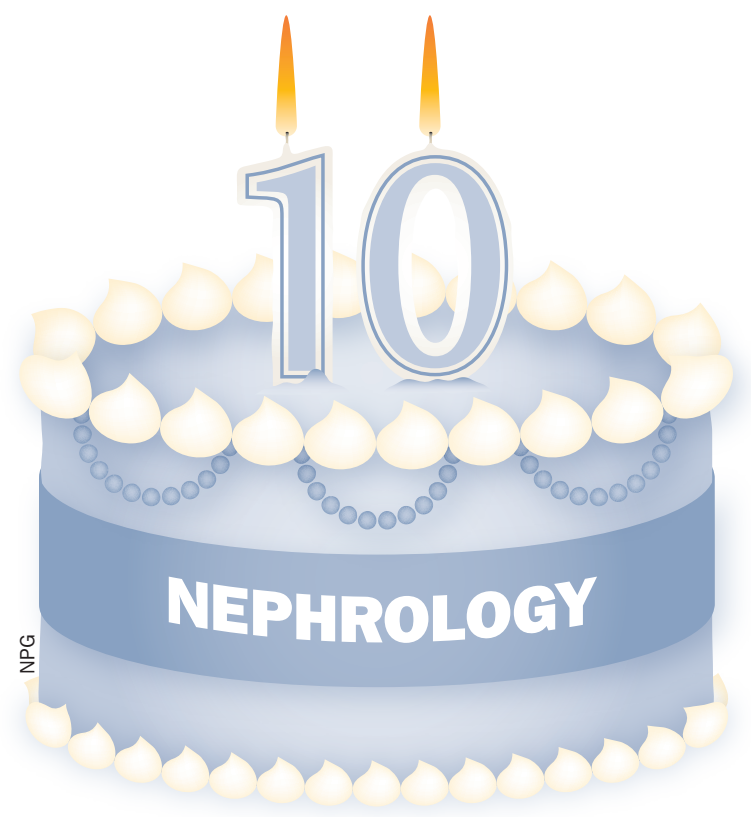

Nephrology has continuously strived to disseminate knowledge with the purpose of advancing research and care of patients with renal disease. We have celebrated some important achievements of the ISN, for example, their successful lobbying of the United Nations to recognize chronic kidney disease (CKD) as an important noncommunicable disease in its own right (Feehally, J. Nat. Rev. Nephrol. 8, 12-13, 2012), and we support efforts to promote global kidney health (Levin, A. \& Perkovic, V. Nat. Rev. Nephrol. 9, 195-197, 2013; Allison, S. J. Nat. Rev. Nephrol. 11, 125, 2015).

In this special issue we celebrate achievements across the field of nephrology. Four Decade in Review articles look back at progress in the broad fields of glomerular disease, genetics of renal disease, acute kidney injury and renal transplantation. In addition, in a Viewpoint article we ask five of our longest-serving Advisory Board members to comment on achievements across the breadth of nephrology and their predictions for the future. As well as highlighting some of the key areas in which progress has been made-for example, in the understanding of podocyte biology and glomerular disease, hypertension, polycystic kidney disease, and the increasingly recognized contribution of genetics to renal disease-they offer their opinions on roadblocks to success. As described by these experts, such barriers include the identification and use of appropriate end points in clinical trials, the complexity and multimorbidity of CKD, the infrequency of some renal diseases and the lack of sensitive and specific biomarkers to appropriately profile patients and individualize care.

Overcoming these barriers will be essential to progress the field and provide effective treatments for patients with renal disease. Some barriers will not be as difficult to overcome as others; for example, large collaborative studies are increasingly being used to enable larger sample sizes for the study of rare diseases. Communication and collaboration will also be essential to more accurately characterize complex phenotypes-a necessary step if the field is to achieve its goal of personalized medicine for patients with renal disease.

In the coming years, Nature Reviews Nephrology will continue to provide expert insight, discussion, and context to advances in nephrology. We would like to take this opportunity to extend our gratitude to everyone who has contributed to the success of the journal-advisors, authors, reviewers and readers. We look forward to further collaborations to advance nephrology research and ignite the interest of those new to the field.

doi:10.1038/nrneph.2015.163

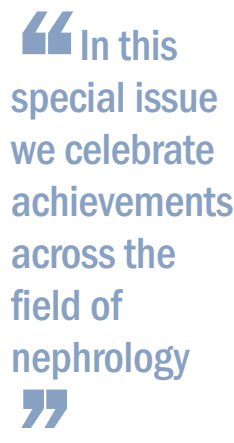

Susan J. Allison is the Chief Editor of Nature Reviews Nephrology.

Competing interests The author declares no competing interests. 